\title{
IRS 13N: a new comoving group of sources at the Galactic center
}

\author{
K. Mužić ${ }^{1,2}$, R. Schödel ${ }^{3}$, A. Eckart ${ }^{1,2}$, L. Meyer ${ }^{4}$, and A. Zensus ${ }^{2,1}$ \\ 1 I. Physikalisches Institut, Universität zu Köln, Zülpicher Str. 77, 50937 Köln, Germany \\ e-mail: muzic@ph1.uni-koeln.de \\ 2 Max-Planck-Institut für Radioastronomie, Auf dem Hügel 69, 53121 Bonn, Germany \\ 3 Instituto de Astrofísica de Andalucía, Camino Bajo de Huétor 50, 18008 Granada, Spain \\ ${ }^{4}$ University of California, Division of Astronomy and Astrophysics, Los Angeles, CA 90095-4705, USA
}

Received 25 July 2007 / Accepted 26 February 2008

ABSTRACT

\begin{abstract}
Context. The Galactic center IRS 13E cluster is located 3.2" from SgrA*. It is an extremely dense stellar association containing several Wolf-Rayet and O-type stars, at least four of which show a common velocity. Only half an arcsecond north of IRS 13E there is a complex of extremely red sources so-called IRS 13N. Their nature is still unclear. Based on analysis of their colors, there are two main possibilities: (1) dust-embedded sources older than few Myr or (2) extremely young objects with ages less than 1 Myr.

Aims. We present the first proper motion measurements of IRS 13N members and then give proper motions of four of IRS 13E stars resolved in the $L^{\prime}$-band.

Methods. The $L^{\prime}$-band $(3.8 \mu \mathrm{m})$ observations were carried out using the NACO adaptive optics system at the ESO VLT. Proper motions were obtained by linear fitting the stellar positions extracted by StarFinder as a function of time, weighted by positional uncertainties.

Results. We show that six of seven resolved northern sources show a common proper motion, thus revealing a new comoving group of stars in the central half parsec of the Milky Way. The common proper motions of IRS 13E and IRS 13N clusters are significantly $(>5 \sigma)$ different. We also performed a fitting of the positional data for those stars onto Keplerian orbits, assuming SgrA* as the center of the orbit. Our results favor the very young stars hypothesis.
\end{abstract}

Key words. Galaxy: center - infrared: stars - astrometry - open clusters and associations: indvidual: IRS 13N open clusters and associations: individual: IRS 13E

\section{Introduction}

The central half parsec of the Milky Way is host to a surprisingly high number of massive young stars (see e.g. Paumard et al. 2006; Ghez et al. 2005), organized in at least one disk-like structure of clockwise rotating stars (CWS; Genzel et al. 2003; Levin \& Beloborodov 2003; Paumard et al. 2006). Paumard et al. (2006) also propose the existence of a second, less populated disk of counter-clockwise rotating stars (CCWS). The mechanism responsible for the presence of young stars in the strong tidal field of the super-massive black hole $(\mathrm{SMBH})$ at the position of $\mathrm{SgrA}^{*}$ is not clear. Two most prominent scenarios include star formation "in-situ" (in an accretion disk; Levin \& Beloborodov 2003; Nayakshin et al. 2006), and the inspiral of a massive stellar cluster formed at a safe distance of 5-30 pc from the Galactic Center (GC; Gerhard 2001; McMillan \& Portegies Zwart 2003; Kim et al. 2004; Portegies Zwart et al. 2006). At this point, the former scenario seems to be favored by number of authors (Nayakshin \& Sunyaev 2005; Nayakshin et al. 2006; Paumard et al. 2006). Also, recent results by Stolte et al. (2007) definitively rule out the possibility that the Arches cluster could migrate inwards and fuel the young stellar population in the GC.

A special challenge and a good observational test for both the above mentioned scenarios is provided by the existence of the IRS 13 group of sources. IRS 13E (located 3 " west and $\sim 1.5^{\prime \prime}$ south of $\left.\mathrm{SgrA}^{*}\right)$ is the densest stellar association after the stellar cusp in the immediate vicinity of SgrA* and contains several massive Wolf-Rayet (WR) and O-type stars (Maillard et al. 2004; Paumard et al. 2006; Moultaka et al. 2005). It is generally considered to be associated with the mini-spiral material (Moultaka et al. 2005; Paumard et al. 2004) and is probably bound, since four out of seven identified stars show highly correlated velocities (Maillard et al. 2004; Schödel et al. 2005). For both of the above mentioned scenarios of star formation at the GC there are several issues when dealing with IRS 13E. In principle, such a cluster could have been formed in an accretion disk (Milosavljević \& Loeb 2004; Nayakshin \& Cuadra 2005). However, in numerical simulations of star forming disks, fragmentation of a disk cannot produce such a dominant feature (Nayakshin et al. 2007). In light of the cluster in-fall scenario, an intermediate-mass black hole (IMBH) was proposed to reside in the center of the cluster (Maillard et al. 2004). The existence of an IMBH makes the process of cluster in-spiral much more efficient in terms of increasing dynamical friction and thus allowing the most massive stars that reside in the center of a very massive $\left(>10^{6} M_{\odot}\right)$ stellar cluster to reach the central parsec of the Galaxy within their lifetimes (Hansen \& Milosavljević 2003; Berukoff \& Hansen 2006; Portegies Zwart et al. 2006; but see Kim et al. 2004, for a characterization of the problems with this hypothesis). However, the presence of the IMBH in IRS $13 \mathrm{E}$ is disputed. Schödel et al. (2005) analyze the velocity dispersion of cluster stars and derive that the mass of such an object should be at least $7000 M_{\odot}$, making its existence implausible. Both the 
X-ray (Baganoff et al. 2003) and radio (Zhao \& Goss 1998) source at the position of IRS $13 \mathrm{E}$ can be explained by colliding winds of high-mass losing stars (Coker et al. 2002; Zhao \& Goss 1998), without the need for any unusual object. Paumard et al. (2006) suggest that, in the case that IRS 13E is associated with the Bar of the mini-spiral, the mass of the stellar content would be high enough to keep the cluster from disruption.

A second comoving group of stars, called IRS 16SW and located $1.9^{\prime \prime}$ in projection from $\mathrm{SgrA}^{*}$, was reported by $\mathrm{Lu}$ et al. (2005). Four of the five members of the IRS 16SW comoving group are spectroscopically identified as young massive stars, indicating ages comparable to those of the IRS 13E cluster. However, unlike IRS 13E, IRS 16SW shows only a slight stellar number density enhancement.

Approximately $0.5^{\prime \prime}$ north of IRS 13E, a small cluster of unusually red compact sources has been reported (IRS $13 \mathrm{~N}$; Eckart et al. 2004). Eight sources have been resolved and labeled $\alpha$ through $\eta$ as shown in Figs. 1 and 2 of Eckart et al. (2004). A strong infrared excess is due to the emission of warm ( $T \sim 1000 \mathrm{~K}$ ) dust (Moultaka et al. 2005). The authors propose two possible explanations for the nature of IRS 13N: (1) objects older than few Myr and similar to bow-shock sources reported by Tanner et al. (2005) or (2) extremely young stars (0.1-1 Myr old). The latter scenario implies more recent star formation than what has been assumed so far for the GC environment.

Here we present first proper motion measurements of the IRS $13 \mathrm{~N}$ sources and additionally present proper motions of four of IRS 13E sources, including one for which no proper motion has previously been published. All proper motions were derived using $L^{\prime}$-band NACO/VLT images ${ }^{1}$.

\section{Observations and data reduction}

The $L^{\prime}(3.8 \mu \mathrm{m})$ images were taken with the NAOS/CONICA adaptive optics assisted imager/spectrometer (Lenzen et al. 1998; Rousset et al. 1998; Brandner et al. 2002) at the UT4 (Yepun) at the ESO VLT. The data set includes images from 6 epochs (2002.66, 2003.36, 2004.32, 2005.36, 2006.41 and 2007.39) with a resolution of $\sim 100$ mas and a pixel scale of 27 mas/pixel. Data reduction (bad pixel correction, sky subtraction, flat field correction) and formation of final mosaics was performed using the DPUSER software for astronomical image analysis (Ott; see also Eckart \& Duhoux 1990). All the images were deconvolved using the linear Wiener filter. The absolute positions of sources in our AO images were derived by comparison to the VLA positions of IRS 10EE, 28, 9, 12N, 17, 7 and $15 \mathrm{NE}$ as given by Reid et al. (2003).

Stellar positions were extracted with StarFinder (Diolaiti et al. 2000) and transformed into the common coordinate system with the aid of 19 reference stars. The positions of the reference stars were corrected for the stellar proper motions as derived from the $K_{\mathrm{S}}$-band images (see Mužić et al. 2007). In Mužić et al. (2007) we have shown that the lower resolution $L^{\prime}$-band data can be reliably used to obtain proper motions.

Proper motions were derived by linear fitting of positions as a function of time, weighted by the positional uncertainties. Both the error of the transformation to the reference frame and the error of the stellar position fitting contribute to the uncertainties.

We assume the distance to the GC to be $7.6 \mathrm{kpc}$ (Eisenhauer et al. 2005).

\footnotetext{
${ }^{1}$ Based on observations collected at the European Southern Observatory, Chile.
}

Table 1. Proper motions of IRS $13 \mathrm{~N}$ sources observed in $L^{\prime}$-band.

\begin{tabular}{lllrr}
\hline \hline Name & $\Delta \alpha^{a}$ & $\Delta \delta^{a}$ & $v_{\mathrm{RA}}^{b}$ & $v_{\text {Dec }}^{b}$ \\
\hline$\alpha$ & -2.70 & -1.48 & $44 \pm 8$ & $147 \pm 13$ \\
$\beta$ & -2.89 & -1.25 & $-6 \pm 20$ & $228 \pm 15$ \\
$\gamma$ & -3.10 & -0.99 & $-114 \pm 14$ & $298 \pm 7$ \\
$\delta$ & -2.92 & -0.85 & $-33 \pm 13$ & $248 \pm 9$ \\
$\epsilon$ & -2.88 & -1.02 & $-58 \pm 15$ & $306 \pm 13$ \\
$\zeta$ & -3.16 & -0.82 & $-134 \pm 9$ & $333 \pm 6$ \\
$\eta$ & -3.11 & -0.66 & $-91 \pm 22$ & $323 \pm 39$ \\
$13 \mathrm{~N}^{c}$ & -3.01 & -0.93 & $-73 \pm 7$ & $289 \pm 8$ \\
\hline
\end{tabular}

${ }^{a}$ Relative to $\mathrm{SgrA} *$, in arcseconds, ${ }^{b}$ all velocities are in $\mathrm{km} \mathrm{s}^{-1}$; the uncertainties represent the $1 \sigma$ uncertainty of the linear fit, ${ }^{c}$ average proper motion of six comoving sources $\beta-\eta$.

Table 2. Proper motions of IRS 13E sources observed in $L^{\prime}$-band.

\begin{tabular}{lcrrr}
\hline \hline Name & $\Delta \alpha^{a}$ & $\Delta \delta^{a}$ & $v_{R A}^{b}$ & $v_{\text {Dec }}^{b}$ \\
\hline E1 & -2.95 & -1.64 & $-107 \pm 11$ & $-118 \pm 6$ \\
E2 & -3.16 & -1.73 & $-228 \pm 8$ & $53 \pm 9$ \\
E3 & -3.17 & -1.53 & $-158 \pm 4$ & $71 \pm 3$ \\
E5 & -3.39 & -1.53 & $-175 \pm 45$ & $140 \pm 72$ \\
$13 E^{c}$ & -3.17 & -1.61 & $-167 \pm 12$ & $37 \pm 18$ \\
$13 E^{d}$ & -3.24 & -1.60 & $-187 \pm 15$ & $88 \pm 24$ \\
\hline
\end{tabular}

${ }^{a}$ Relative to $\mathrm{SgrA}^{*}$, in arcseconds, ${ }^{b}$ all velocities are in $\mathrm{km} \mathrm{s}^{-1}$; the uncertainties represent the $1 \sigma$ uncertainty of the linear fit, ${ }^{c}$ average proper motion of four IRS 13E sources, ${ }^{d}$ average proper motion of IRS 13E without E1.

\section{Results}

\subsection{Proper motions}

In Tables 1 and 2 we list proper motions of IRS 13N and IRS 13E sources identified in $L^{\prime}$-band, respectively. In Fig. 1 we show proper motions of all stars superposed on the $L^{\prime}$-band image. Stars $\beta$ through $\eta$ show similar proper motions, revealing a new comoving group of sources at the GC. As shown in Eckart et al. (2004), only $\eta$ can also be detected in the $K$-band. Paumard et al. (2006) give the full velocity information for this star: $v_{\mathrm{RA}}=(-52 \pm 28) \mathrm{km} \mathrm{s}^{-1}, v_{\text {Dec }}=(257 \pm 28) \mathrm{km} \mathrm{s}^{-1}$ and $v_{\mathrm{r}}=(40 \pm 40) \mathrm{km} \mathrm{s}^{-1}$. The authors identify it as a member of a possibly existing second stellar disk (CCWS).

Concerning IRS 13E, the obtained proper motion for E1 shows the biggest discrepancy with respect to previously published data (Paumard et al. 2006; Schödel et al. 2005). It also deviates significantly from the proper motions of the other three stars. It was already noted that E1 shows the largest deviation from the systemic proper motion of the cluster (Schödel et al. 2005). Paumard et al. (2006) argue that this star is possibly not bound to the cluster. This is supported by our result.

It is important to note that, in addition to a very dense stellar population in the region, a large amount of diffuse dust emission detected at $3.8 \mu \mathrm{m}$ makes the astrometry more challenging to perform than at shorter wavelengths. For this reason, StarFinder was not able to resolve all the previously reported $K$-band sources in the region. E3 is known to be at least a double source, while here we present it as a single one. E4 is located close to E3 but is much fainter in the $L^{\prime}$-band. Therefore it could not be clearly identified by StarFinder. However, we were able to resolve the faint source E5 in all epochs and present the first proper motion measurement of this star. E5 was proposed to be a dusty WR star (Maillard et al. 2004), and it seems to exhibit a 

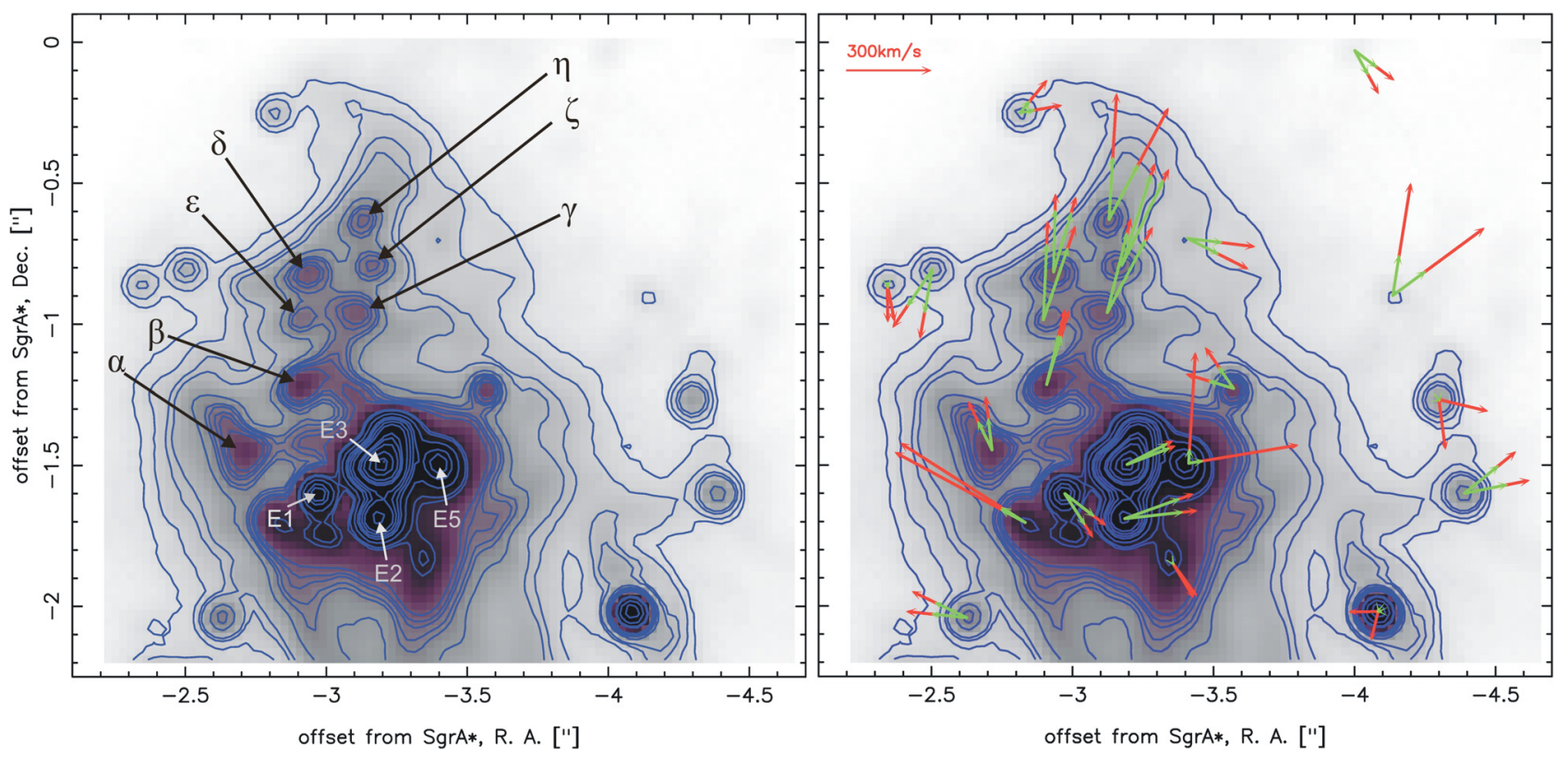

Fig. 1. Identification and proper motions of stars in IRS 13 complex. Four arrows are shown for each source, indicating the $\pm 3 \sigma$ uncertainty of the value and direction of its proper motion.

proper motion consistent with those of E2 and E3. This implies that it may be a cluster member.

From Tables 1 and 2 one can see that the average proper motions of the two clusters are significantly $(>5 \sigma)$ different. Thus, it seems that two clusters do not belong to the same system.

\subsection{Keplerian orbit fitting}

In order to analyze our measurements we attempted to fit the positional data of IRS $13 \mathrm{~N}$ stars showing similar proper motions ( $\operatorname{stars} \beta$ to $\eta$ ) to Keplerian orbits, assuming that the gravitational potential is dominated by the SMBH at the position of SgrA*. For details of the fitting procedure and definition of parameters see e.g. Eisenhauer et al. (2003). We assumed the distance and the mass of SgrA* to be $7.6 \mathrm{kpc}$ and $3.6 \times 10^{6} M_{\odot}$, respectively. The velocity of SgrA* was assumed to be zero (Reid \& Brunthaler 2004). Under the assumption that the stars are really on bound orbits around SgrA*, it is clear that our data cover only a small part of such an orbit. We also lack the radial velocity information for all of the stars. As a consequence, the $\chi^{2}$ minimization gives a very wide span of orbital parameters for which $\chi^{2}<\chi_{\min }^{2}+1$, even when fixing most of the free parameters (the mass of and the distance to SgrA*, as well as the position and the velocity of the center of the orbit). Therefore we repeated the orbital fitting for each star introducing the radial velocity data as given in Paumard et al. (2006) for $\eta$. We varied the inclination angle $i$ between $0^{\circ}$ and $90^{\circ}$ in steps of $1^{\circ}, \Omega$ (position angle of the ascending node) between $0^{\circ}$ and $180^{\circ}$ in steps of $20^{\circ}$ and $\omega$ (longitude of periastron) between $0^{\circ}$ and $360^{\circ}$ with a step size of $20^{\circ}$. The results are given in Table 3 . We list the best fit parameters $i$ and $\Omega$, and give the $1 \sigma$ range for the inclination. We also list the range in eccentricities $(e)$ and semi-major axes $(a)$ resulting from the fits for which there is some combination of angles $(i, \Omega, \omega)$ that gives a fit better than $1 \sigma\left({ }^{2}\right)$. For all the stars except $\zeta$, the best fit reduced $\chi^{2}$ value ranges between 0.6 and 1.5 . For $\zeta$ the best fit is obtained with $\chi^{2}=3.9$, probably

\footnotetext{
${ }^{2}$ A fit with $\chi^{2}<\chi_{\min }^{2}+1$, for a corresponding star. For a convenience, we refer to all orbits that satisfy this condition as $1 \sigma$ orbits.
}

implying that either positional uncertainties are underestimated, or that assuming the radial velocity of $\eta$ is a poor assumption. In the last three lines of Table 3 we give the best-fit parameters for the Keplerian orbits fitted onto the average positions of the stars within the IRS13N and IRS13E comoving groups. For IRS 13E we perform the fitting procedure twice: first including the star E1 and afterwards considering it a non-member.

Paumard et al. (2006) conclude that IRS 13E is on a fairly eccentric orbit, with $e \gtrsim 0.5$, which we confirm. For all IRS $13 \mathrm{~N}$ stars, with the exception of $\beta$, the range in eccentricities of $1 \sigma$ orbits is restricted to fairly low values $(<0.4)$, in agreement with the Paumard et al. (2006) value listed for $\eta$. Note that the corresponding semi-major axes also result fall within a restricted range of values. In contrast, both parameters ( $e$ and $a$ ) are poorly constrained for the $\operatorname{star} \beta$. The size of the best fit orbit of $\beta$ is an order of magnitude higher than those of the other five stars. Since the orbit of $\beta$ is radically different from orbits of all the other stars, we note that it probably should not be regarded as part of the co-moving group. Therefore we do not show its orbit in Fig. 2. Since $\eta$ is the only star with known radial velocity, we took its orbital parameters and attempted to fit other stars onto the same orbit. We note that it is not possible to fit all other stars onto exactly the same orbit (same $i, \Omega$ and $\omega$ ). Therefore we fix $i$ and $\Omega$ and let $\omega$ vary. This confines the stars to the same plane, within which they can still have different orbits. For three stars $(\gamma, \epsilon, \zeta)$ there exist $1 \sigma$ orbits with given $i$ and $\Omega$, the star $\delta$ can be reasonably (but still not within $1 \sigma$ ) fitted, and $\beta$ fails completely to be fitted onto this plane (Fig. 2b). To get a feeling about the uncertainties of our analysis, we plot several $1 \sigma$ orbits for the same star $(\eta)$ in Fig. 2c.

The implications of these results are further discussed in Sect. 4.2.

\section{Discussion}

\subsection{3-dimensional position}

Paumard et al. (2004) report the morphology and radial velocities of the mini-spiral gas based on $\mathrm{He} \mathrm{I}$ and $\mathrm{Br} \gamma$ observations. 

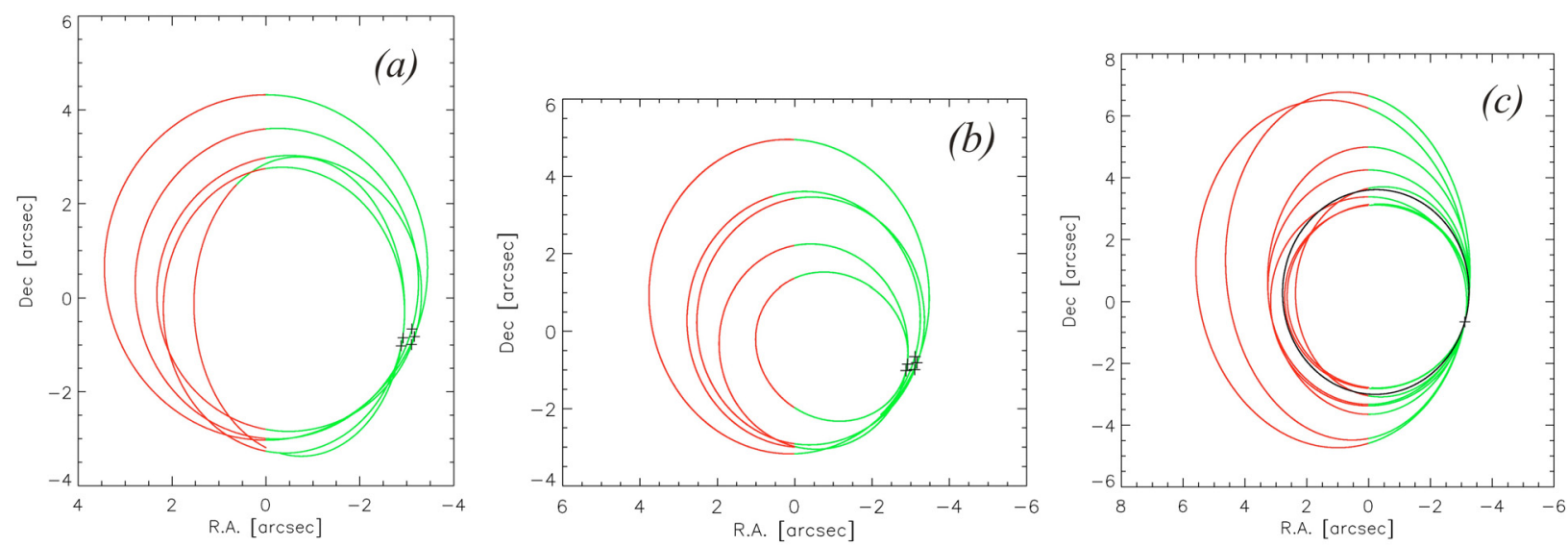

Fig. 2. a) the best fit orbital solutions for five IRS $13 \mathrm{~N}$ stars $(\gamma-\eta)$; b) orbital solutions for a single plane $\left(i=24^{\circ}, \Omega=180^{\circ}\right)$, for stars $\gamma-\eta$; c) chosen $1 \sigma$ orbits for $\eta$, with the best fit orbit colored black; NOTES: part of the orbit in front of the plane of the sky is colored red; crosses mark present positions of the stars; axes show the offset from SgrA*.

Table 3. Results of the Keplerian orbit fitting.

\begin{tabular}{cccccc}
\hline \hline Name & $i\left(^{\circ}\right)^{a} \Omega\left({ }^{\circ}\right)^{a}$ & $i\left(^{\circ}\right)^{b}$ & $e$ & $a\left({ }^{\prime \prime}\right)$ \\
\hline$\beta$ & 79 & 180 & $53-80$ & $0-0.9$ & $4.0-180.0$ \\
$\gamma$ & 19 & 180 & $4-67$ & $0.17-0.21$ & $2.6-4.0$ \\
$\delta$ & 48 & 180 & $39-71$ & $0.15-0.4$ & $2.7-15.0$ \\
$\epsilon$ & 33 & 180 & $17-56$ & $0.1-0.3$ & $2.5-7.2$ \\
$\zeta$ & 18 & 180 & $0-30$ & $0.1-0.3$ & $3.2-4.7$ \\
$\eta$ & 24 & 180 & $1-45$ & $0.1-0.3$ & $2.8-5.7$ \\
${ }_{\text {IRS 13N }}^{d}$ & 28 & 180 & $14-49$ & $0.1-0.2$ & $2.8-5.7$ \\
IRS 13E & 83 & 100 & $80-85$ & $0.5-0.9$ & $12.5-145.0$ \\
IRS 13E & 70 & 120 & $14-42 ; 58-80$ & $0.5-0.8$ & $2.3-66.0$
\end{tabular}

${ }^{a}$ Best fit parameters $\left(\chi^{2}=\chi_{\min }^{2}\right),{ }^{b}$ all inclination values for which $\chi^{2}<$ $\chi_{\min }^{2}+1,{ }^{c}$ calculated for $(i, \Omega, \omega)$ combinations for which $\chi^{2}<\chi_{\min }^{2}+1$, ${ }^{d}$ average orbit, ${ }^{e}$ including E1, ${ }^{f}$ not including E1.

At the position of the IRS 13 sources, the Northern Arm and the Bar of the mini-spiral are overlapping along the line of sight, with the Bar being placed further away from the observer. The authors suggest that IRS 13E must be either embedded in the Bar, or very close to it. Also, the radial velocities of the IRS 13E stars are similar to the radial velocities of the Bar material at this position (Paumard et al. 2004). Spectroscopic observations of the entire IRS 13 region (Moultaka et al. 2005) indicate a close spatial correlation between the stellar sources and the surrounding material. Higher water ice and hydrocarbon absorptions in the northern part of IRS 13, as well as a redder continuum emission, suggest that the IRS $13 \mathrm{~N}$ sources are more embedded in the ISM than the rest of the complex. The radial velocity of $\eta$ is consistent with the radial velocity of the Bar at this position (between 0 and $50 \mathrm{~km} \mathrm{~s}^{-1}$, Paumard et al. 2004). If the northern sources that show similar proper motions also have correlated radial velocities, it would be reasonable to suggest that the entire complex is indeed associated with the Bar. According to our orbital fits, IRS $13 \mathrm{~N}$ is located a few equivalent arcseconds behind Sgr A*, which nicely agree with the view of Paumard et al. (2004) about the Bar being located somewhat behind Sgr A*. This would imply that the IRS $13 \mathrm{E}$ and IRS $13 \mathrm{~N}$ stellar complexes are spatially close. The existence of two comoving clusters, each moving in a completely different direction, poses a lot of challenges for star formation scenarios at the GC.

\subsection{Is IRS $13 \mathrm{~N}$ a bound system?}

As can be seen from the orbital analysis, the best fit orbits for IRS $13 \mathrm{~N}$ stars have rather different orbital parameters. Observing six stars on different orbits exactly at the moment when they appear to be very close in projection and to have similar proper motions is not very probable. There is no single orbit onto which all of the stars would fit with a good $\chi^{2}$, but at least four of the stars can be confined to the same plane. In this case the stars are again on different orbits and consequently have different orbital periods, spanning the range from 1000 to 3000 years. This again implies that the present arrangement is temporary. Also, it is curious that the moment at which we observe them coincides exactly with the presence of dense gas at their position. Interestingly, their most likely common plane seems to coincide with the plane of the counter-clockwise moving stars (CCWS; Paumard et al. 2006). The existence of the CCWS disk is still a matter of debate. According to Paumard et al. (2006), the disk is sparsely populated (12 stars), with $\eta$ belonging to it. The assumption that all IRS $13 \mathrm{~N}$ stars belong to the CCWS significantly increases the population of the disk, and at the same time weakens the claim that the CCWS is essentially in non-circular motion. In the analysis of Paumard et al. (2006), seven out of twelve CCWS stars are on eccentric orbits $(e>0.4)$, three of those belonging to IRS 13E system. We find that five of the IRS $13 \mathrm{~N}$ stars are on low-eccentricity orbits.

The analysis of $1 \sigma$ orbits for one star (Fig. 2c) shows that the uncertainty of semi-major axes $(a)$, as well as that of orbital periods $(P)$, is of the same order as the differences between $a$ and $P$ of each star. Therefore, at this point we still cannot exclude that IRS $13 \mathrm{~N}$ stars are indeed orbiting together. In the following we consider the possibility that the cluster is bound. We calculate the velocity dispersion of stars as

$$
\sigma^{2}=\sigma_{\mathrm{pm}}^{2}-\sum_{i=1}^{N}\left[\operatorname{error}^{2}\left(v_{x, i}\right)+\operatorname{error}^{2}\left(v_{y, i}\right)\right] /[2(N-1)]
$$

where $\sigma_{\mathrm{pm}}$ is the dispersion of the measured proper motions and the second term removes the influence of the proper motion measurement uncertainties.

The mass estimate from the velocity dispersion of stars gives $\sim 3300 M_{\odot}$, an unrealistically high mass for such a cluster. For a comparison, we note that Paumard et al. (2006) estimate the total stellar mass of IRS $13 \mathrm{E}$ to be $\sim 1000 M_{\odot}$. We note that even if 
the uncertainties in proper motion measurements were underestimated, the final mass of the cluster calculated from the velocity dispersion would remain high. For instance, a hypothetical underestimation of our uncertainties of $30 \%$ would result in $\sim 8 \%$ lower velocity dispersion and the mass of $2700 M_{\odot}$.

The Hill radius

$r_{\mathrm{Hill}} \approx a(1-e)\left(\frac{m}{3 M}\right)^{\frac{1}{3}}$

for a cluster of $m=3300 M_{\odot}$, with $a=(2.8-5.7)^{\prime \prime}$ and $e=0.1-$ 0.3 is $r_{\text {Hill }} \approx 0.17^{\prime \prime}-0.34^{\prime \prime}$, with the average value remarkably close to the observed cluster radius of $\sim 0.25^{\prime \prime}$. The other way around, if we set $r_{\text {Hill }} \sim 0.25^{\prime \prime}$, the maximum $a$ and minimum $e$, the minimum mass needed to keep the cluster from disruption would be $1250 M_{\odot}$.

The relaxation time for a system of $N$ stars with the mean mass $m_{*}$ is given by

$t_{\text {relax }} \approx \frac{N}{8 \ln \Lambda} \times t_{\text {cross }}$

where $t_{\text {cross }}=R / v$ and $\Lambda=R \mathrm{v}^{2} / G m_{*} \approx N$ (Binney \& Tremaine 1987). Also, it is important to note that tidal interactions tend to shorten relaxation timescales. A cluster with the IRS $13 \mathrm{~N}$ velocity dispersion would have a $t_{\text {relax }}$ of the order of the IRS $13 \mathrm{~N}$ orbital period only if it was consisted of 500-1000 stars. For $m \sim 3300 M_{\odot}$ contained in 500-1000 main sequence stars we get that stars should be as bright as $m_{K}=17-18$ (assuming the extinction $A_{K} \approx 3$, Schödel et al. 2007). These stars would be observed. For a lower mass limit of $1250 M_{\odot}$, we get $m_{K}=19-$ 21 , exactly at the limit of NACO $K$-band data. Therefore it seems that the system could hardly be bound and survive for a significant time in the orbit around SgrA*.

If IRS $13 \mathrm{~N}$ stars are not bound, the observed velocity dispersion probably indicates that the cluster is in the process of dissolution. The velocity dispersion will subsequently diminish the stellar surface number density of the cluster, which would within only a few hundred years reach the background value given by Schödel et al. (2007).

\subsection{The nature of IRS $13 \mathrm{~N}$}

The detailed discussion about the nature of IRS $13 \mathrm{~N}$ sources is presented in Eckart et al. (2004). Among other possibilities, the authors argue that infrared excess sources IRS $13 \mathrm{~N}$ could represent objects that have colors and luminosities consistent with YSOs and Herbig Ae/Be stars. Dusty envelopes that usually surround those objects would then give rise to the observed strong infrared excess. It is striking that six of the sources that are very close in projection, also show very similar proper motion values. In the previous section we argue that IRS $13 \mathrm{~N}$ could hardly survive in the present arrangement for a significant amount of time. The indication of a dynamically young stellar system concurs with the Eckart et al. (2004) hypothesis of IRS 13N stars being extremely young objects. But why then does this object exsist at all? Timescales for its disruption seem to be much shorter than any timescales within which stars normally form. Here we must take into account that the Galactic Center is an extremely complex environment and that any star formation process that takes place there is completely different from the "normal" star formation via the fragmentation of a molecular cloud. Timescales for star formation could be significantly shorter. The free-fall time in a collapsing cloud at the distance of the young stars in the central half parsec around $\mathrm{Sg} \mathrm{rA} *$ is of the order of 100-1500yr. Also, simulations of star formation in a gaseous disk around Sgr A* by Nayakshin et al. (2007) indicate that the timescales for star formation are fairly short, of the order of several thousand years. This is comparable to the orbital timescale of IRS 13N.

A serious obstacle for forming stars at the GC is that the gas densities in the central parsec are way too low for the gas to be able to form stars. Even the highest density estimates for the circumnuclear disk (CND; Christopher et al. 2005) are several orders of magnitude lower than required to fulfill the criterion for Jeans instability. However, with the aid of shocks and collisions, a small clump of gas in-falling to the center could be highly compressed and star formation within it triggered. As a matter of fact, the mini-spiral is a short-lived feature $\left(t_{\text {dyn }} \sim 10^{4} \mathrm{yr}\right)$ and is infalling right now. Furthermore, the material of the mini-spiral is susceptible to shocks, as discussed by e.g. Mužić et al. (2007). A possibility of forming stars in small associations has never been discussed so far for this region. Star formation in small groups definitely would not be able to account for the young co-eval stellar population in the central parsec, but could, in general, be possible and result in small associations like IRS 13N.

However, it is clear that we still must be very careful when drawing a conclusion that IRS $13 \mathrm{~N}$ cluster contains very young stars. In case that the cluster is indeed a part of the CCWS disk and that the stars were formed simultaneously with other CCWS stars, then they must be several Myr old. The fact that they are apparently strongly extincted may then indicate that they are similar to the mini-spiral bow-shock sources (Tanner et al. 2005), but many times less massive and luminous (see discussion in Eckart et al. 2004). The question remains: why are they clustered?

\section{Conclusions}

In this paper we present the proper motion measurements of IRS $13 \mathrm{~N}$, a newly discovered co-moving group of red sources at the GC. We discuss the boundness of a cluster and a possibility that these stars are the youngest stellar object ever observed in this environment. Ongoing star formation in the GC would have significant implications on our understanding of this particular environment, and star formation in galactic nuclei in general. It has been assumed that the star formation in the GC occurred in two star bursts, $\sim 100 \mathrm{Myr}$ and $\sim 7 \mathrm{Myr}$ ago (Krabbe et al. 1995). The existence of objects younger than 1 Myr would imply that in-situ star formation in the GC and near massive black holes in general is possible. Furthermore, this would also mean that star formation in the GC must not necessarily be related to star bursts and that, in fact, a continuous star formation could go on.

The question of the real nature of the IRS $13 \mathrm{~N}$ cluster remains open. More imaging and especially high resolution spectroscopic data that could reveal features attributed to YSOs will be necessary to confirm our hypothesis that the stars are extremely young.

Acknowledgements. We thank the referee, Dr. M. Morris, for comments and suggestions that improved this work. Part of this work was supported by the Deutsche Forschungsgemeinschaft (DFG) via SFB 494. K. Mužić and L. Meyer were supported for this research through a stipend from the International Max Planck Research School (IMPRS) for Radio and Infrared Astronomy at the Universities of Bonn and Cologne.

\section{References}

Baganoff, F. K., Maeda, Y., Morris, M., et al. 2003, ApJ, 591, 891 Berukoff, S. J., \& Hansen, B. M. S. 2006, ApJ, 650, 901 
Binney, J., \& Tremaine, S. 1987, Galactic Dynamics (Princeton University Press) Brandner, W., Rousset, G., Lenzen, R., et al. 2002, Messenger, 107, 1

Christopher, M. H., Scoville, N. Z., Stolovy, S. R., \& Yun, M. S. 2005, ApJ, 622, 346

Coker, R., Pittard, J. M., \& Kastner, J. H. 2002, A\&A, 383, 568

Diolaiti, E., Bendinelli, O., Bonaccini, D., et al. 2000, A\&AS, 147, 335

Eckart, A., \& Duhoux, P. R. M. 1990, in Astrophysics with Infrared Arrays (San Francisco: ASP), ed. R. Elston, ASP Conf. Ser., 2, 14, 336

Eckart, A., Moultaka, J., Viehmann, T., Straubmeier, C., \& Mouawad, N. 2004, ApJ, 602, 760

Eisenhauer, F., Schödel, R., Genzel, R., et al. 2003, ApJ, 597, L121

Eisenhauer, F., Genzel, R., Alexander, T., et al. 2005, ApJ, 628, 246

Genzel, R., Schödel, R., Ott, T., et al. 2003, ApJ, 594, 812

Gerhard, O. 2001, ApJ, 546, L39

Ghez, A. M., Salim, S., Hornstein, S. D., et al. 2005, ApJ, 620, 744

Hansen, B. M. S., \& Milosavljević, M. 2003, ApJ, 593, L77

Kim, S. S., Figer, D. F. \& Morris, M. 2004, ApJ, 607, L123

Krabbe, A., Genzel, R., Eckart, A., et al. 1995, ApJ, 447, L95

Lenzen, R., Hofmann, R., Bizenberger, P., \& Tusche, A. 1998, in Infrared Astronomical Instrumentation, ed. A. M. Fowler, Proc. SPIE, 3354, 606

Levin, Y., \& Beloborodov, A. 2003, ApJ, 590, L33

Lu, J. R., Ghez, A. M., Hornstein, S. D., Morris, M., \& Becklin, E. E. 2005, ApJ, 625, L51

Maillard, J. P., Paumard, T., Stolovy, S. R., \& Rigaut, F. 2004, A\&A, 423, 155
McMillan, S. L. W., \& Portegies Zwart, S. F. 2003, ApJ, 596, 314

Milosavljević, M., \& Loeb, A. 2004, ApJ, 604, L45

Moultaka, J., Eckart, A., Schödel, R., Viehmann, T., \& Najarro, F. 2005, A\&A, 443,163

Mužić, K., Eckart, A., Schödel, R., Meyer, L., \& Zensus, A. 2007, A\&A, 469, 993

Nayakshin, S., \& Cuadra, J. 2005, A\&A, 437, 437

Nayakshin, S., \& Sunyaev, R. 2005, MNRAS, 364, L23

Nayakshin, S., Dehnen, W., Cuadra, J., \& Genzel, R. 2006, MNRAS, 366, 1410

Nayakshin, S., Cuadra, J., \& Springel, V. 2007, MNRAS, 379, 21

Paumard, T., Maillard, J.-P., \& Morris. 2004, A\&A, 426, 81

Paumard, T., Genzel, R., Martins, F., et al. 2006, ApJ, 643, 1011

Portegies Zwart, S. F., Baumgardt, H., McMillan, S. L. W., et al. 2006, ApJ, 641, 319

Reid, M. J., \& Brunthaler, A. 2004, ApJ, 616, 872

Reid, M. J., Menten, K. M., Genzel, R., et al. 2003, ApJ, 587, 208

Rousset, G., Lacombe, F., Puget, P., et al. 1998, in Adaptive Optical System Technologies, ed. D. Bonaccini, Proc. SPIE, 3353, 516

Schödel, R., Eckart, A., Alexander, T., et al. 2007, A\&A, 469, 125

Schödel, R., Eckart, A., Iserlohe, C., Genzel, R., \& Ott, T. 2005, ApJ, 625, L111

Stolte, A., Ghez, A. M., Morris, M., et al. 2007, [arXiv:0706.4133v1]

Tanner, A., Ghez, A. M., Morris, M. R., \& Christou, J. C. 2005, ApJ, 624, 742

Zhao, J.-H., \& Goss, W. 1998, ApJ, 499, L163 